\title{
The black hole spins of quasars
}

\author{
Bei You ${ }^{1,2}$ and Xinwu Cao ${ }^{1}$ \\ ${ }^{1}$ Key Laboratory for Research in Galaxies and Cosmology, Shanghai Astronomical \\ Observatory, Chinese Academy of Sciences, 80 Nandan Road, Shanghai, 200030, China \\ email: youbeiyb@gmail.com \\ ${ }^{2}$ University of Chinese Academy of Sciences, 19A Yuquanlu, Beijing 100049, China
}

\begin{abstract}
We present the estimates of the black hole spins of five quasars. The peaks of the spectra of the accretion discs surrounding massive black holes in quasars are in the far-UV or soft X-ray band, which are usually not observed. However, in the disc corona model, the soft photons from the disc are Comptonized to high energy in the hot corona, and the hard X-ray spectra (luminosity and spectral shape) contain the information of the incident spectra from the disc. The values of black hole spin parameter $a$ are inferred from the spectral fitting, which spread over a large range, $\sim-0.94$ to 0.998 .
\end{abstract}

Keywords. galaxies: nuclei, galaxies: kinematics and dynamics, methods: numerical

\section{Introduction}

The black hole play an important role on the energetic radiation (Kormendy \& Richstone 1995) and the production of the relativistic jets/outflows (Blandford \& Znajek 1977) for both X-ray binaries and active galactic nuclei (AGN). The relativistic jets/outflows are important for the evolution of the host galaxy including the star formation (Fabian 2012). Therefore better understanding of these processes requires the measurements of the black hole spins.

One reliable method to constrain the black hole spins of X-ray binaries is the continuum fitting (Zhanget al. 1997). In principle, this method can be used to constrain the black hole spins for AGNs. However, the application of this method in AGNs meets a major difficulty, i.e., the spectral peaks of accretion discs surrounding massive black holes with $\sim 10^{8} M_{\odot}$ are in the far-UV or soft X-ray band, which are unobservable for most AGNs.

In the accretion disk corona model, a portion of the soft photons of the spectral peaks originating from the disc undergo Comptonization in the hot corona, and the scattered photons are dominantly in the hard X-ray band (You et al. 2012). This implies that the observed hard X-ray spectra (luminosity and spectral shape) contain the information of the incident spectra from the cold disc. It seems possible to constrain the values of black hole spin parameter $a$ in AGNs with a general relativistic accretion disc corona model, even if the spectral peaks of the accretion discs are not observed.

\section{Results}

Using the relativistic accretion disc corona model (see You et al. 2012; You 2014), we fit the observed optical/UV "Big Blue Bump" (BBB) at $\sim 10^{15}-10^{16} \mathrm{~Hz}$ and the power-law hard X-ray continuum spectra of five quasars from Shanget al. (2011). The left tail of the BBBs can be observed in infared/optical/UV wavebands, which provide useful information of the BBBs.

There are four free parameters in our model calculations, i.e., the mass accretion rate $\dot{m}$, the spin parameter $a$, the power fraction $f$ dissipated in the corona, and the viewing 
Table 1. The fitting parameters of the sources

\begin{tabular}{lllllll}
\hline Object & $\log M$ & $f$ & $a$ & $\dot{m}$ & $\theta$ & $T_{e}(\mathrm{keV})$ \\
\hline PG 1322+659 & 8.29 & $0.47_{-0.12}^{+0.10}$ & $-0.94_{-0.06}^{+0.14}$ & $0.388_{-0.020}^{+0.019}$ & $25.7_{-4.7}^{+3.5}$ & $265.3_{-32.1}^{+32.1}$ \\
PG 1115+407 & 8.18 & $0.41_{-0.03}^{+0.06}$ & $-0.55_{-0.15}^{+0.15}$ & $0.420_{-0.005}^{+0.020}$ & $10.0_{-3.1}^{+7.3}$ & $241.2_{-65.5}^{+65.5}$ \\
4C 10.06 & 9.00 & $0.60_{-0.10}^{+0.15}$ & $0.7_{-0.05}^{+0.05}$ & $0.415_{-0.017}^{+0.020}$ & $39.4_{-2.0}^{+2.2}$ & $194.7_{-15.7}^{+15.7}$ \\
4C 39.25 & 9.33 & $0.47_{-0.02}^{+0.03}$ & $0.998_{-0.004}^{+0.000}$ & $0.344_{-0.011}^{+0.008}$ & $42.5_{-1.4}^{+0.9}$ & $158.3_{-4.4}^{+4.4}$ \\
OS 562 & 8.92 & 0.33 & $0.96_{-0.04}^{+0.03}$ & $0.505_{-0.020}^{+0.027}$ & $13.0_{-9.6}^{+7.2}$ & $142.5_{-8.4}^{+8.4}$ \\
\hline
\end{tabular}

Note. The black hole mass $M$ is taken from Tanget al. (2012). $T_{e}$ is the maximum value of the electron temperature of the corona, which is calculated with the best fitted values of free parameters $a$ and $\dot{m}$.

Figure 1. The spectral fitting results for $4 \mathrm{C} 39.25$. The radio and near infrared data are not used in our accretion disc corona model fittings. The photometric points marked as blue filled circles are used for fitting. The solid black line represents the power law X-ray spectrum in $2-10 \mathrm{keV}$. The red line represents the best fitting result. The black hole masses $M$ of these five quasars are given in Tanget al. (2012), which are estimated with the broad-line width and the scaling relationships. The spectral fitting results for other sources can be found in You (2014).

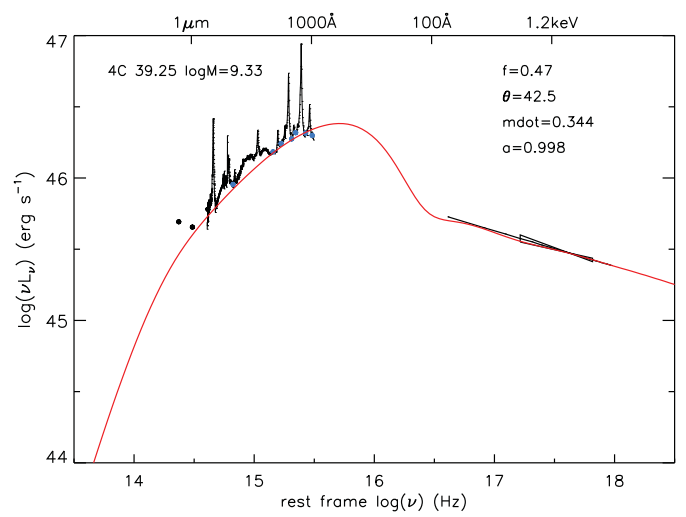

angle $\theta$ with respect to disc axis. We find that the fraction $f$ is mainly determined by the X-ray spectral index $\Gamma$, which is almost independent of the values of other parameters. With the derived value of $f$ from $\Gamma, \dot{m}$ and $\theta$ are constrained with the luminosity and spectral shape in optical waveband (the red tail of the BBB). These two parameters are almost independent of the black hole spin parameter a, because the emission in this waveband is dominantly from the outer region of the disc. Finally, the black hole spin parameter $a$ is constrained mainly with the observed hard X-ray luminosity.

\section{Acknowledgements}

This work is supported by the NSFC (grants 11173043, 11121062, 11233006, 11073020, and 11373056), the Fundamental Research Funds for the Central Universities (WK2030220004), the CAS/SAFEA International Partnership Program for Creative Research Teams (KJCX2YW-T23), and Shanghai Municipality.

\section{References}

Blandford R. D. \& Znajek R. L., 1977, MNRAS, 179, 433

Fabian, A. C. 2012, ARAA, 50, 455

Kormendy J. \& Richstone D., 1995, ARA $\& A$, 33, 581

Shang Z., et al., 2011, ApJS, 196, 2

Tang B., Shang Z., Gu Q., Brotherton M. S., \& Runnoe J. C., 2012, ApJS, 201, 38

You, B., Cao, X., \& Yuan, Y.-F. 2012, ApJ, 761, 109

You, B., Cao, X., \& Yuan, Y.-F. 2014, arXiv:1401.5659

Zhang S. N., Cui W., \& Chen W., 1997, ApJ, 482, L155 